\title{
Entering the spotlight: hepatitis B surface antigen-specific B cells
}

\author{
Christoph Neumann-Haefelin and Robert Thimme \\ Department of Medicine II, University Medical Center Freiburg, Faculty of Medicine, University of Freiburg, Freiburg, Germany.
}

\begin{abstract}
Hepatitis B virus-specific (HBV-specific) T cells have been identified as main effector cells in HBV clearance. In contrast, B cells producing neutralizing antibodies against the HBV surface antigen (HBsAg) have been studied in little detail, mainly due to methodical limitations. In this issue of the $J C I$, two reports use a new technique to specifically detect and characterize $\mathrm{HBsAg-}$ specific B cells ex vivo. Indeed, these cells are present, but show phenotypic alterations and impaired function during acute and chronic HBV infection. Thus, HBsAg-specific B cells are a novel attractive target for antiviral strategies toward functional cure of chronic HBV infection.
\end{abstract}

\section{Hepatitis B surface antibody in history and treatment}

A fourth of the world's population has been infected with hepatitis B virus (HBV), and an estimated 250 million people are chronically infected, putting them at risk for progressive liver disease, liver cirrhosis, liver failure, and hepatocellular carcinoma. Of adults infected with HBV, 95\% clear the virus spontaneously, while $90 \%$ of newborns develop chronic infection. Since HBV infection does not induce an interferon response, HBV is a stealth virus with respect to the innate immune system (1, 2). Therefore, HBV clearance depends on a successful adaptive immune response. Indeed, a multispecific and vigorous $\mathrm{HBV}$-specific $\mathrm{CD}^{+} \mathrm{T}$ cell response has been associated with viral clearance $(3,4)$, while $\mathrm{HBV}$-specific $\mathrm{CD}^{+} \mathrm{T}$ cell exhaustion is a hallmark of persistent infection (5-7). Less attention, however, has been paid to the role of neutralizing antibodies targeting the envelope protein of $\mathrm{HBV}$, the HBV surface antigen (HBsAg). Indeed, the hepatitis B surface antibody (anti-HBs) is only detectable in the blood of HBV-infected individuals after the loss of HBsAg, either upon the resolution of acute infection or in case of the rare event of spontaneous or drug-induced clearance of chronic HBV infection. Thus, anti-HBs is seen as not playing a major role in $\mathrm{HBV}$ clearance. Anti-HBs, on the other hand, has been established as a diagnostic tool, indicating protective immunity after acute-resolving HBV infection or vaccination as well as "functional cure" of chronic HBV infection (HBsAg to anti-HBs seroconversion). There are, however, several clinical observations that argue against such a minor role of anti-HBs. First, antiHBs contained in hepatitis B immunoglobulin (HBIG) is well known to prevent vertical $\mathrm{HBV}$ transmission from infected mothers to newborns as well as reinfection of liver transplants in previously HBVinfected hosts. Second, B cell-depleting drugs used in oncology and rheumatology, such as the anti-CD20 antibody rituximab, induce $\mathrm{HBV}$ reactivation in $\mathrm{HBV}$ carriers and even in patients with "resolved" HBV in whom nuclear HBV cccDNA persists as a viral reservoir for decades (8). These clinical observations indicate that antiHBs has a more prominent role than previously acknowledged. Indeed, anti-HBs can block HBV cell entry, either by block-

Related Articles: pp. 4573 and 4588

Conflict of interest: The authors have declared that no conflict of interest exists.

Reference information: / Clin Invest. 2018;128(10):4257-4259. https://doi.org/10.1172/JCI124098. 
A

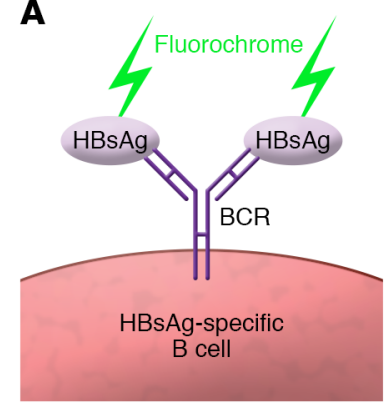

B

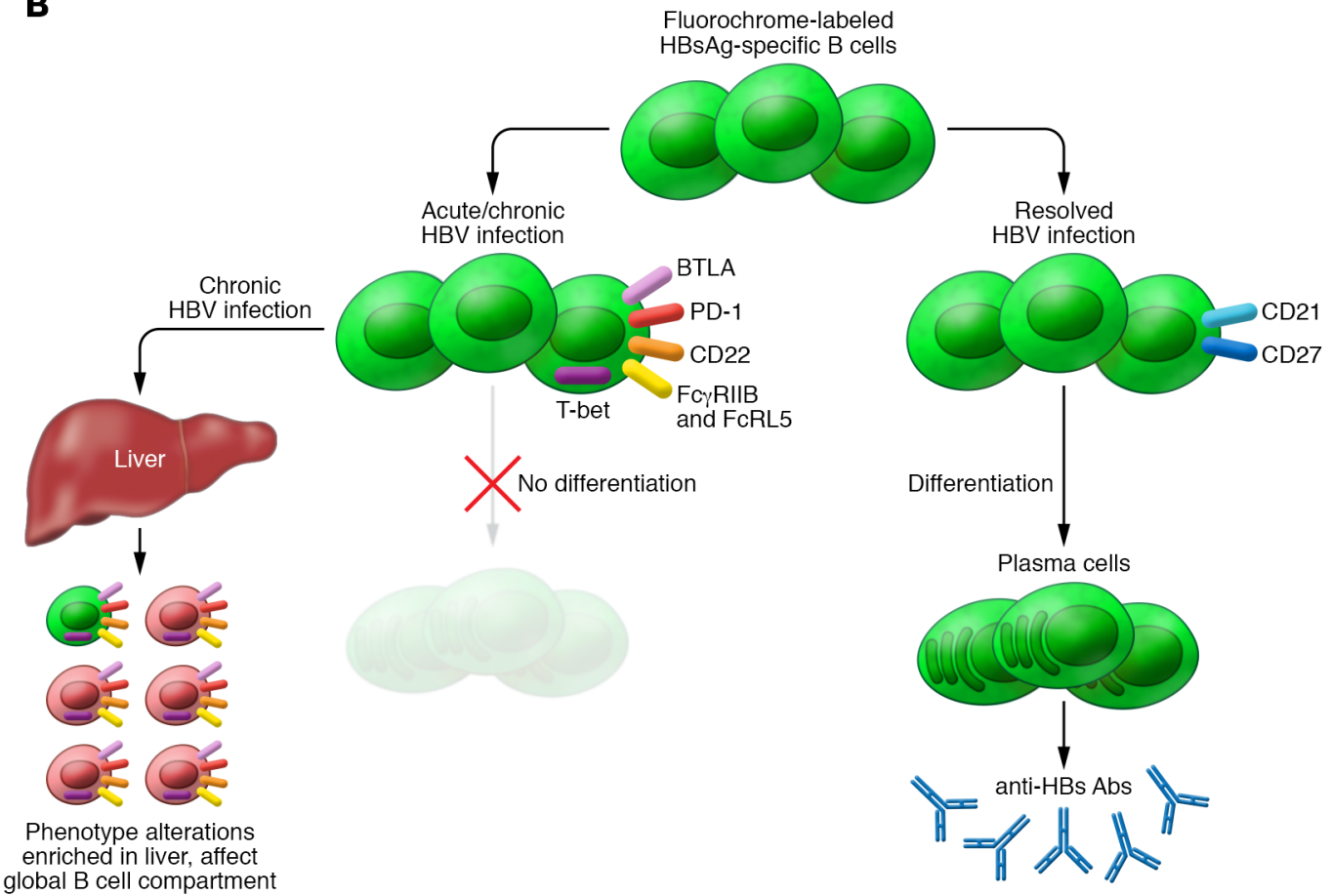

Figure 1. HBsAg-specific B cells in HBV infection. (A) Fluorochrome-labeled HBsAg binds to the HBsAg-specific B cell receptor (BCR) of HBsAg-specific B cells, allowing their detection and characterization directly ex vivo. Using this approach, phenotypical as well as functional differences between $\mathrm{HBsAg-}$ specific B cells in acute and chronic infection versus resolved infection were identified. (B) In acute and chronic infection, HBsAg-specific B cells are present in similar frequencies compared with their presence in resolved infection; however, they display low expression of CD21 and CD27 (consistent with atypical memory B cells) as well as a high expression of inhibitory receptors, such as PD-1, BTLA, CD22, Fc $\gamma$ RIIB, and FcRL5. They also highly express the transcription factor T-bet and are not able to differentiate into anti-HBs-secreting plasma cells. These phenotypical alterations are enriched in the liver. However, they are not restricted to HBsAg-specific B cells, but affect the global B cell compartment of patients with acute or chronic HBV infection.

Bertoletti laboratory (13) and Burton et al. from the Maini laboratory (14) addressed the issue of lack of anti-HBs during acute and chronic HBV infection by making use of a new technique to detect HBsAgspecific B cells. Specifically, they utilized fluorochrome-labeled recombinant HBsAg as "bait" to detect and characterize HBsAg-specific B cells by FACS analysis ex vivo. Strikingly, by using a similar approach independently, the two groups came to the very same results (Figure 1): first, HBsAg-specific B cells are present in acute, chronic, and resolved HBV infection in similar frequencies. Second, HBsAg-specific B cells isolated from patients with acute as well as chronic, but not resolved, HBV infection are unable to mature into anti-HBs-secreting cells in vitro. Third, in phenotypical analyses, these HBsAg-specific B cells in acute and chronic HBV infection resemble "atypical memory B cells" that are characterized by low expression of CD21 and CD27, but high expression of inhibitory markers, such as PD-1, and the transcription factor
T-bet (15). Fourth, Burton et al. demonstrated that these HBsAg-specific atypical memory B cells accumulate in the liver, the site of infection and inflammation of HBV-infected patients. Fifth, this phenotype and functional impairment are not restricted to HBsAg-specific B cells, but also affect the global B cell population in HBV-infected patients, a finding with so far unclear implications that needs further clarification. Sixth, the function of HBsAgspecific $B$ cells can be partially restored in vitro by specific culture conditions, such as PD-1 blockade $(13,14)$ or the addition of IL-2, IL-21, and CD40 ligand-expressing (CD40L-expressing) feeder cells (13).

\section{Opening the doors for $\mathrm{B}$ cell- targeted research in hepatitis $B$} These results clearly argue for $\mathrm{B}$ cell dysfunction rather than antibody depletion as the main reason for the lack of anti-HBs in acute and chronic HBV infection. Thus, these findings also open the door for $\mathrm{B}$ cells as targets of novel antiviral strategies aiming at a functional cure of chronic HBV infection (seroconversion from HBsAg to anti-HBs). Indeed, none of the currently developed antiviral strategies, including viral entry inhibitors, viral capsid release inhibitors, immune modulators, or therapeutic vaccination, achieve functional cure in a substantial number of patients, even if used in combination with the currently approved antiviral compounds pegylated IFN- $\alpha$ and nucleoside/nucleotide analogs, such as tenofovir or entecavir (16). It is important to note that the current studies by Salimzadeh and Le Bert et al. and Burton et al. are not the completion, but just the beginning of $B$ cell-targeted research in the field of hepatitis $\mathrm{B}$, similar to the emerging $\mathrm{T}$ cell research after the broad introduction of peptide/MHC tetramers nearly two decades ago (17). Indeed, many open issues remain, such as the cellular and molecular mechanisms leading to HBsAg-specific B cell dysfunction, e.g., the involvement of impaired follicular helper $\mathrm{T}$ (Tfh) cells $(18,19)$ and the mechanisms and (clinical) consequences of impairment of the global B cell population as well as 
the optimal strategy for restoring HBsAgspecific B cell function on the way to functional cure of chronic HBV infection. Of note, PD-1 blockade enhances antiviral B cell immunity against SIV in macaques in vivo (20). Specifically, it will be important to identify the best intervention for the restoration of B cell function, e.g., targeting of single versus multiple inhibitory receptors or choice of the most promising cytokine and feeder cell to be used in vivo and/or for adoptive cell transfer. With these important questions and exciting opportunities, HBsAg-specific B cells are expected to be in the spotlight for the next several years.

\section{Acknowledgments}

This work is supported by the Deutsche Forschungsgemeinschaft: SFB1160, Immunemediated pathology as a consequence of impaired immune reactions (IMPATH), project 8 (to $\mathrm{RT}$ ) and project 10 (to $\mathrm{CNH}$ ); and TRR-179, Determinants and dynamics of elimination versus persistence of hepatitis virus infection, project 1 (to RT) and project 2 (to $\mathrm{CNH}$ ).

Address correspondence to: Christoph Neumann-Haefelin, University Hospital Freiburg, Department of Medicine II, Hugstetter Strasse 55, 79106 Freiburg, Germany. Phone: 49.761.270.32800; Email: christoph. neumann-haefelin@uniklinik-freiburg.de.
1. Mutz P, et al. HBV bypasses the innate immune response and does not protect $\mathrm{HCV}$ from antiviral activity of interferon. Gastroenterology. 2018;154(6):1791-1804.e22.

2. Wieland S, Thimme R, Purcell RH, Chisari FV. Genomic analysis of the host response to hepatitis B virus infection. Proc Natl Acad Sci US A. 2004;101(17):6669-6674.

3. Bertoletti A, Ferrari C. Adaptive immunity in HBV infection. J Hepatol. 2016;64(1 suppl):S71-S83.

4. Thimme R, et al. CD8(+) T cells mediate viral clearance and disease pathogenesis during acute hepatitis B virus infection. J Virol. 2003;77(1):68-76.

5. Bengsch B, Martin B, Thimme R. Restoration of HBV-specific CD $8^{+} \mathrm{T}$ cell function by PD-1 blockade in inactive carrier patients is linked to T cell differentiation. J Hepatol. 2014;61(6):1212-1219.

6. Fisicaro $P$, et al. Targeting mitochondrial dysfunction can restore antiviral activity of exhausted HBV-specific CD8 T cells in chronic hepatitis B. Nat Med. 2017;23(3):327-336.

7. Schurich A, et al. Distinct metabolic requirements of exhausted and functional virusspecific CD8 T cells in the same host. Cell Rep. 2016;16(5):1243-1252.

8. Loomba R, Liang TJ. Hepatitis B reactivation associated with immune suppressive and biological modifier therapies: current concepts, management strategies, and future directions. Gastroenterology. 2017;152(6):1297-1309.

9. Corti D, Benigni F, Shouval D. Viral envelopespecific antibodies in chronic hepatitis B virus infection. Curr Opin Virol. 2018;30:48-57.

10. Maruyama T, McLachlan A, Iino S, Koike K, Kurokawa K, Milich DR. The serology of chronic hepatitis B infection revisited. JClin Invest. 1993;91(6):2586-2595.
11. Oliviero B, et al. Enhanced B-cell differentiation and reduced proliferative capacity in chronic hepatitis $\mathrm{C}$ and chronic hepatitis $\mathrm{B}$ virus infections. J Hepatol. 2011;55(1):53-60.

12. Xu X, et al. Reversal of B-cell hyperactivation and functional impairment is associated with HBsAg seroconversion in chronic hepatitis B patients. Cell Mol Immunol. 2015;12(3):309-316.

13. Salimzadeh L, et al. PD-1 blockade partially recovers dysfunctional virus-specific $B$ cells in chronic hepatitis B infection. J Clin Invest. 2018;128(10):4573-4587.

14. Burton AR, et al. Circulating and intrahepatic antiviral B cells are defective in hepatitis B. J Clin Invest. 2018;128(10):4588-4603.

15. Portugal S, Obeng-Adjei N, Moir S, Crompton PD, Pierce SK. Atypical memory B cells in human chronic infectious diseases: An interim report. Cell Immunol. 2017;321:18-25.

16. Testoni B, Durantel D, Zoulim F. Novel targets for hepatitis B virus therapy. Liver Int. 2017;37(suppl 1):33-39.

17. Maini MK, et al. Direct ex vivo analysis of hepatitis B virus-specific CD8(+) T cells associated with the control of infection. Gastroenterology. 1999;117(6):1386-1396.

18. Raziorrouh B, et al. Virus-specific CD $4^{+} \mathrm{T}$ cells have functional and phenotypic characteristics of follicular T-helper cells in patients with acute and chronic HCV infections. Gastroenterology. 2016;150(3):696-706.e3.

19. Wang X, et al. Dysregulated response of follicular helper $\mathrm{T}$ cells to hepatitis b surface antigen promotes HBV persistence in mice and associates with outcomes of patients. Gastroenterology. 2018;154(8):2222-2236.

20. Velu V, et al. Enhancing SIV-specific immunity in vivo by PD-1 blockade. Nature. 2009;458(7235):206-210. 